DESIGN AND USE OF THE EMBRITTLEMENT DATA BASE (EDB)

F. W. Sta11mann

Oak Ridge National Laboratory

Oak Ridge, Tennessee 37831
CONF-870501--4

DE87 010372

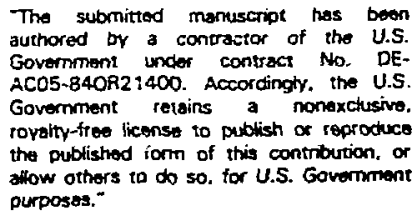

purposes."

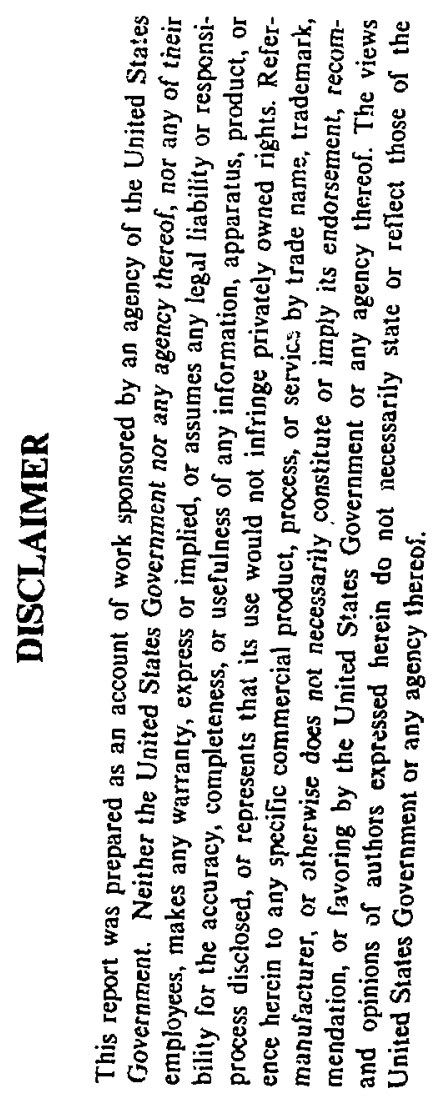

*Prepared for the

U.S. Nuclear Regulatory Commission Office of Nuclear Regulatory Research Washington, D.C. 20555

under Interagency Agreement 0551-0551-A1

NRC FIN No. B0415

Prepared by the

Oak Ridge National Laboratory

Oak Ridge, Tennessee 37831

operated by

MARTIN MARIETTA ENERGY SYSTEMS, INE.

for the

U.S. DEPARTMENT OF ENERGY

under Contract No. DE-AC05-84OR2 1400 


\title{
DESIGN AND USE OF THE EMBRITTLEMENT DATA BASE (EDB)
}

\author{
F. W. Stallmann* \\ Oak Ridge National Laboratory \\ Oak Ridge, Tennessee 37831
}

\begin{abstract}
The architecture of the Embrittlement Data Base (EDB) is described. This data base contains a comprehensive collection of experimental data related to irradiations of reactor pressure vessel steels in surveillance capsules and test reactors. Software is being developed for easy retrieval and analysis of the data. Data and software will be made available to interested parties on a cooperative basis.
\end{abstract}

REY WORDS: embrittlement, data base, surveillance capsules, material irradiation

\footnotetext{
* Research sponsored by Office of Nuclear Regulatory Research, U.S. Nuclear Regulatory Commission under Interagency Agreement 0551-0551-Al with the U.S. Department of Energy under contract DE-AC05-840R21400 with the Martin Marietta Energy Systems, Inc.
} 
DESIGN AND USE OF THE EMBRITTLEMENT DATA BASE (EDB)

\section{BACKGROUND}

The neutron radiation in operating nuclear power reactors causes embrittlement of the pressure vessel and support structures. This embrittlement diminishes the resistance against crack extensions which may be initiated during routine heat-up and cool-down operations and that are aggravated by more severe pressurized thermal shock conditions caused by loss of cooling (LOC) accidents. The Embrittlement Data Base (EDB) has been established to collect radiation embrittlement data as reported from a large variety of test reactor irradiations and surveillance capsules for many different steel plates, forgings, and weldments. These data can be used to validate theoretical radiation embrittlement models and to establish licensing requirements associated with pressurized thermal shock, end-of-life, and life extension considerations.

Data collections for embrittlement studies have been available for some time, in particular from the Materials Property Council (MPC) ${ }^{1}$ and the Electric Power Research Institute (EPRI).2,3 The difference between these data collections and the EDB described in this paper is that the former are "static" in the sense that they are configured in a predetermined data format that requires a complete redesign if data needs and availabilities are changing. The design of the EDB, on the other hand, is "dynamic" in that it readily accommodates changing requirements for input data and user requests for specific combinations of output data. This is made possible through the use of the data base managemert tools, specifically dBASE III $^{4}$ by Ashton Tate in connection with the IBM-PC/AT. 
Such a syster is more than adequate for the purposes of the EDB and is widely available so that data can be easily exchanged between interested parties in the form of diskettes.

The data collection started with the MPC Data Base. These data were traced to the original reports and checked for consistency and duplication. Raw experimental data were added as available and efforts were made to determine uncertainties. The main effort at present is to extend the collection to experiments that were not included in the original data base and also to fill the gaps. Cooperation is sought with vendors, laboratories, and government agencies, not only in the U.S. but on an international basis. The availability of a truly comprehensive data base with information from many and varied sources will, in the long run, benefit everybody and it is hoped that the reluctance of some parties to shäre proprietory data can be overcome.

\section{DATA BASE ARCHIZECTURE}

The EDB consists of a collection of many data files, each of which is tailored to a particular type of data. No particular restrictions are imposed $\underline{a}$ priori as to the size and format except that of the DOS for PCs and the dBASE III PLUS software, when applicable. The basic philosophy is that the software has to be determined by the given data structure and not the other way around. This implies that a considerable effort has to go into the development and maintenance of the software. However, this effort and expense will always be tiny compared to the expense of creating the data, and good software will also reduce considerably the time and effort for the final inalysis. 
The EDB file collection consists of three different sets of files:

1. raw data files,

2. evaluated data files, and

3. processed data files.

The data flow through these files is indicated in Fig. 1.

The raw data files contain the collection of input data, essentially as reported and collected without regard to their eventual use. Each data item in a raw data file is identified as to the source document including page number and all source documents are collected in a reference file. Included in the raw data files are evaluations of the reporting laboratory such as estimates of fluences or shifts of NDT obtained from raw Charpy data. No attempts are made to correct the reported data except for obvious transcription errors or duplications. Details of the raw data files will be discussed in the next section.

The evaluated data files form the core of the EDB. In these files, those data from the raw data files that are of prime importance for embrittlement studies are extracted, checked for consistency and correctness, and uncertainty estimates are added. The transition from raw data to evaluated files may also involve some processing such as taking averages of several independent: measurements or to obtain transition temperature and upper shelf values from raw Charpy data. Details will be given in Section 4. From the evaluated files, any desired combination of data can be obtained as a "processed file." Since the evaluated files are given in dBASE III format, the standard dBASE III software can be 


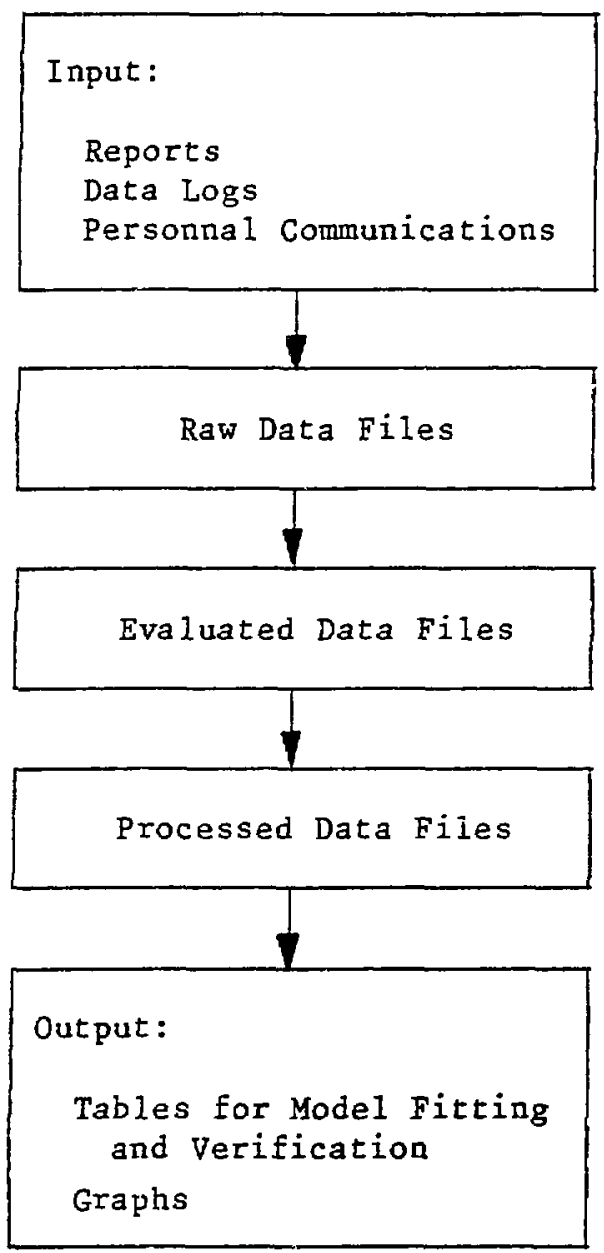

Fig. 1. Data Flow in EDB. 
used to generate the desired processed files. Further steps consist of fitting data to particular models and graphic displays.

A11 raw data and evaluated files are related to each other through certain key identifiers. These identifiers refer in a unique manner to a particular piece of material and, for lata on irradiated specimens, to the reactor and the experimental or surveillance capsule. A unique identification is also provided for each report or other reference that provides the input for the raw data files. The keys are the following:

\section{a. Material Key}

This key identifies the particular material by heat number or similar identification and, if applicable, by location of specimen within source material and its direction relative to the rolling or welding direction.

b. Environment Rey

This key specifies the reactor and the experiment, including, if necessary, the location of the specimen within the experimental capsule. This may also include information about annealing and re-irradiation, when applicable.

c. Reference Key

Each document from which data are obtained is characterized by a unique key and all data in raw data files can be traced to their source by these keys. Page numbers are included where applicable. 


\section{DATA COLLECTION AND INPUT REQUIREMENTS}

All data for the EDB are first collected in raw data files. The purpose of these files is to preserve as much as feasible of the details in the experimental results without being restricted by consideration of their eventual use (or non-use). Data format and measurement units are also not critical at this stage since reformatting and conversions can be handled by software. It will be desirable, however, that for future experiments, data are submitted directly in machine-readable form and in a standardized format to the EDB from the participating laboratory. Development and updating of reporting standards, consistent with current practices, is part of the scope of the EDB maintenance.

All data are collected as reported with reference, including page number, attached. Only one data set is included for identical data reported in more than one publication. However, if data are corrected or modified in subsequent publications, both the original and the modified version will be included, properly identified. The collection of raw data is a continuing and accumulative process and only a relatively small percentage of the available data has been collected so far. Types of raw data files that are being collected or considered for future collection are listed below.

a. Reactor environment (data are identified by Environment Keys)

(1) Calculated fluence spectra

(2) Reactor history

(3) Dosimetry

(4) Irradiation temperatures with indication whether obtained 
from melt wires or thermocouples

(5) Junmary as supplied by the original evaluator and/or subsequent re-evaluators

- Eluence $>1.0 \mathrm{MeV}$

- Fluence $>0.1 \mathrm{MeV}$

- dpa

- Thermal fluence

- Fluence rate

- Effective full power time

Fluences and irradiation temperatures are supposed to refer to the capsule center (or other suitable reference point). Specimen-to-specimen variations, if known, are included in data concerning postirradiation properties.

b. Preirradiation properties (data are identified by Material Reys)

(1) Material production

- Material type (e.g., plate, forging, weld, HAZ, and ASTM and other characterizarions such as A302, A508)

- Thickness

- Supplier

- Plant providing heat treatment if different from supplier

- Description of heat treatment as detailed as warranted

In addition for welds:

- Type of weld (e.g., submerged arc)

- Description of stress relief

- Flux type and lot

- Company which fabricated the weld 
- Material ID (or IDs) of the base material that was welded together

In addition for heat effected zone (HAZ)

- Material ID of the weld

- Material ID of the base metal

(2) Chemistry

(3) Raw Charpy data

(4) Raw tensile data

(5) Compact tension data

(6) Drop weight, hardness, and other data

(7) Evaluated Charpy data. A summary of the Charpy test data as evaluated by the reporting labcratory consisting of

- Test temperature relative of $41 \mathrm{~J}(30 \mathrm{ft}-\mathrm{lb})$

- Test temperature r lative to $68 \mathrm{~J}(50 \mathrm{ft}-1 \mathrm{~b})$

- Test temperature relative to $89 \mathrm{~mm}$ (35 mil laterial expansion)

- Upper shelf energy

c. Postirradiation properties (data are identified by Material and Environment Keys)

The data files for postirradiation properties contain essentially the same types of data as the preirradiation property files [see No. b, Items (3) to (7)]. Fluence and irradiation temperature for individual specimen sets may be included to account for possible variations within the surveillance or experimental capsule. 
4. DATA EVALUATION

The purpose of the evaluated files is to provide the user with reliable and consistent data sets that are standardized for easy access. Uncertainties are included which reflect the relative credibility of the data. The processing steps to obtain evaluated files from the raw data involve:

- data reduction;

- determination of uncertainties; and

- elimination of erroneous, inconsistent, or unreliable data. In contrast to the raw data files, these files are being updated continually and the updated version will be periodically released. A $\log$ will be kept for all new entries and changes containing the sources of the data and the reason for change. Every user of the $\mathrm{ELB}$ is requested to report any erroneous or questionable data for the necessary corrections.

In-house data analysis to fit the embrittlement data to a variety of damage models will also be performed. In addition to the primary goal, such data analysis provides also one of the best means to ferret out inconsistencies of the data. Although every reasonable effort is made to avoid errors in the data entry and processing, such errors cannot be completely avoided. Careful data analysj.s combined with the ability to trace all data to their source will, hopefully, keep such errors to a minimum. 


\section{SOFTWARE DEVELOPMENT AND FUTURE PLANS}

The development of software to facilitate the use of the EDB is as important as the data collection. This is aiso a continuing effort and only the most basic parts have been complated so far, utilizing prinarily available data base and rext editing software. The next step will be to customize this software to ease the processing and to prevent mistakes as far as possible. One part of che software is concerned with data entry and validation, and with the determination of uncertainties as outlined in Section 4. This software is of no direct interest to the end user but is needed for quality assurance and data integrity during maintenance of the EDB.

There are three areas for software development that are of immediate interest to the end user:

\section{a. Creating customized tables extracted from the EDB}

The evaluated data base is organized in such a manner that any combination of data can be readily extracted by using the dBASE III PLUS software. This includes selection of specific materials and/or conditions, e.g., Iinde 80 welds or specific fluence conditions, and simple mathematical manipulations, like averages. Customized menu-driven procedures are being developed for users that are not familiar with the dBASE III command set.

\section{b. Data fittings and graphs}

General data fitting and graphics software is available but a considerable effort is required to interface this so:tware with either the evaluated or raw data files in the EDB. Several programs of this type have been written and successfully applied to a model proposed by $D$. Pachur. 
c. Tracing of data to its source document

This procedure is the reverse of data entry and will be necessary if data are questionable or if details need to be known that are not included in the EDB. The use of key identifiers makes it possible to trace all data via raw data files to their source document. Automated procedures for this purpose are planned.

User-related software will be made available together with the data files. Suggestions for specific procedures are welcome and will be considered.

\section{EXAMPLE FOR APPLICATION: ANALYSIS OF THE A302B AND A533B}

\section{FEFERENCE MATERIAL}

The surveillance programs of comercial power rectoctors include, in the majority of cases, correlation materials in the surveillance capsules. These are specimens cut from "standard" steel plates that are similar in composition and heat treatment to the base material in the respective reactor pressure vessei. These specimens are supposed to serve as a reference by comparing the radiation embrittlement of the plant-specific material against the correlation material and to detact anomalies in the radiation environment of the surveillance capsules. Little, if any, use has been made so far of the reference materials. The resson is that, in order to use the correlation materials as a reference, the "normal" behavior in regard to fluence, fluence rate, and irradiation temperature must be established first and this requires the collection of data from a sufficient number of irradiations of the 
material at different fluences, fluence rates, and temperatures. These data are readily available in the EDB including raw Charpy data. The raw data were fitted to a model proposed by D. Pachur and the uncertainties determined. From the information for fluence and chemistry, the NDT shift at $41 \mathrm{~J}$ (30 ft-lb) as predicted by the Revision 2 of Reg. Guide 1.99 was calculated and compared with the experimental values. Prediction and experiment agree within experimental uncertainties and no significant bias was detected in the experimental data relative to the Reg. Guide Model. There is considerable data scatter, but deviations are confined within the limits of the Reg. Guide for the majority oi the capsules; whenever a significant deviation between prediction and experiment occurred, a similarly significant deviation in the same direction was observed for the other materials in the capsule; This suggests that the reference materials can indeed be used as indicators for anomalous conditions in surveillance capsules although the deviations are difficult to quantify. Comparison with the same material in test reactors shows larger than predicted embrittlement at high fluences. This suggests that at high fluence rates, saturation of damage occurs at higher filuences and at higher damage levels. Details will be presented in a separate paper. 


\section{REFERENCES}

1. MPC Data Base, assembled irradiated LWR pressure vessel materials data base prepared by the Metals Properties Council, Inc., New York, NY.

2. Irradiated Nuclear Pressure Vessel Steel Data Base, EPRI NP-2428, Electric Power Research Institute, Palo Alto, CA, June 1982 .

3. Nuclear Plant Irradiated Steel Handbook, EPRI NP-4797, Electric Power Research Institute, Palo Alto, CA, September 1986.

4. dBASE III PLUS, software prepared by Ashton-Tate, Phoenix, AZ. 